\title{
Precise calibration of CCD images with a small field of view
}

\section{Application to observations of Phoebe}

\author{
Q. Y. Peng ${ }^{1,2,3,5}$, A. Vienne ${ }^{2}$, Y. B. Han ${ }^{3}$, and Z. L. $\mathrm{Li}^{4,5}$ \\ 1 Jinan University, Guangzhou 510632, PR China \\ e-mail: pengqy@public.guangzhou.gd.cn \\ ${ }^{2}$ Université de Lille, France and Institut de Mécanique Céleste et de Calcul des Éphémérides, UMR 8028 du CNRS, \\ 1 impasse de l'Observatoire 59000 Lille, France \\ 3 National Astronomical Observatories, CAS, Beijing 100012, PR China \\ 4 Yunnan Observatory, National Astronomical Observatories, CAS, Kunming 650011, PR China \\ 5 Joint Laboratory for Optical Astronomy, CAS, Kunming 650011, PR China
}

Received 16 July 2003 / Accepted 19 May 2004

\begin{abstract}
A precise astrometric calibration method is presented for a CCD image with a small field of view. Its detailed computational formulae are given, and its feasibility and accuracy are tested by the observations of both the star and Phoebe, the 9th satellite of Saturn. This new method can also be applicable to other planetary satellites, asteroids and optical counterparts of extragalactic radio sources.
\end{abstract}

Key words. techniques: miscellaneous - planets and satellites: individual: Saturn - astrometry

\section{Introduction}

Long focus telescopes with a CCD receiver are widely used to determine the positions of some natural satellites of major planets, see Harper et al. (1997), Shen et al. (2001), Vienne et al. (2001a) and Peng et al. (2002) for the major Saturnian satellites. Veiga et al. (2000) and Fienga et al. (2002) have published astrometric CCD observations of the 9th satellite of Saturn, Phoebe. It is well known that the astrometric calibration of a CCD image with a small field of view (for example, $\left.5^{\prime} \times 5^{\prime}\right)$ is difficult. Although a number of calibration methods have been attempted, only parts of them are successful. For example, the use of wide double star pairs is now recognized to be not suitable for accurate scale and orientation (Pascu 1996). A cluster was thought to be the best way to determine the scale of CCD receiver, but Colas \& Arlot (1991) found a problem when calibrating the Martian satellites that was explained mainly by the possible flexions of the telescope from the calibration field to the target one. Harper et al. (1997) reported the same problem when calibrating their observations of Saturnian satellites. On the other hand, there are also successful examples. Veiga \& Vieira Martins (1994) developed a method to use the motion of Uranus to determine both scale and orientation. Also, a "Brighter Moon Calibration" method has been widely used though in theory it can be only used for the faint, inner satellites of major planets. The work of Shen et al. (2001) has generalized this calibration method when a contemporary ephemeris of major Saturnian satellites is referred to.
Some newly published literature is by Vienne et al. (2001a), Peng et al. (2002) and Veiga et al. (2003) for Saturnian satellites. The precise calibration for a small CCD field of view obtained with a long focus telescope must be in the area as small as possible that encloses the target object.

For the astrometric observation of the 9th satellite of Saturn, Phoebe, it is obviously not suitable to use either its motion or "Brighter Moon Calibration" to determine the scale and orientation in a small CCD field of view because of its large separation from Saturn, its faintness and slow speed during the observational run. Veiga et al. (2000) and Fienga et al. (2002) determined the position of Phoebe by a two-step routine. First, they calibrated the field of view with the USNO A2.0 stars and obtained Phoebe's position with respect to these stars. Then the systematic zone error existing both in USNO A2.0 and in the first measured position of Phoebe was removed by comparison with other high-quality catalogue or observations.

In this paper, we present an alternative method to calibrate a small CCD field of view. According to the above analysis, a precise calibration could be realized by a series of CCD images obtained between two bright good-quality stars which are very near to the target object in a celestial area as small as possible. We can cover this small celestial area by overlapping frames of CCD images to combine the calibration reference stars with the target object. Then a global resolution is made to obtain the calibration parameters. This paper is arranged as follows: Sect. 2 describes in detail the proposed method and offers all 
formulae; in Sect. 3, the experimental observations are specified; in Sects. 4 and 5 we give all results of the calibration parameters and the measured positions of the tested star and Phoebe; the last section gives the conclusions.

\section{Proposed method}

\subsection{Philosophy, assumption and requirement}

After one takes a series of frames of CCD images to overlap a small celestial area, there are two kinds of approaches to obtain the calibration parameters. One is to reduce the series of images from different small CCD planes (or plates) into a single common plane. In other words, we hope to have an equivalent big image from these overlapped CCD images. The other is to reduce the series of $\mathrm{CCD}$ images from different small planes into a single common sphere, i.e. the celestial sphere. Here, we prefer to use the latter as shown in Fig. 1. In detail, some assumptions and requirements have to be made beforehand.

i) When a long focus telescope with a CCD receiver moves in a small celestial area (for example $30^{\prime} \times 30^{\prime}$ ), each CCD field of view (for example $5^{\prime} \times 5^{\prime}$ or even smaller) can be described by using only two common parameters (scale $\rho$ and orientation $\phi$ ). In other words, when a frame of a CCD image is measured (in pixels), the pixel coordinate of a celestial object is thought to be linearly linked to its corresponding standard coordinate $(\xi, \eta)$. In fact, almost all researchers (for example, Harper et al. 1997; Vienne et al. 2001a; Peng et al. 2002) have found this linear relation suitable for the reduction of the CCD observations of major Saturnian satellites.

ii) Two reference stars $S_{I}$ and $S_{I I}$ with good-quality positions are needed: their positional errors in a catalogue can be ignored. For example, the external errors of the ACT catalogue are less than 10 mas for the stars $V<9$ mag and $15 \sim 40$ mas for the ones with their magnitudes in 9 mag $<$ $V<11$ mag at the epoch $\mathrm{J} 1995.25$ according to Stone \& Harris (2000).

iii) When the telescope moves in the small celestial area, a series of overlapped CCD frames of images can be obtained. In the overlapped area between any two-neighboring CCD frames, only one faint star (called a connection star; its theoretical position is not precise enough) is required.

\subsection{Positional relations in a single frame of CCD image}

We use the following well-known and strict relations to compute a celestial object's standard coordinate $(\xi, \eta)$.

$\xi=\frac{\cos \delta \sin \left(\alpha-\alpha_{\mathrm{c}}\right)}{\sin \delta \sin \delta_{\mathrm{c}}+\cos \delta \cos \delta_{\mathrm{c}} \cos \left(\alpha-\alpha_{\mathrm{c}}\right)}$

$\eta=\frac{\sin \delta \cos \delta_{\mathrm{c}}-\cos \delta \sin \delta_{\mathrm{c}} \cos \left(\alpha-\alpha_{\mathrm{c}}\right)}{\sin \delta \sin \delta_{\mathrm{c}}+\cos \delta \cos \delta_{\mathrm{c}} \cos \left(\alpha-\alpha_{\mathrm{c}}\right)}$

Where $(\alpha, \delta)$ is its theoretical position from a catalogue, $\left(\alpha_{\mathrm{c}}, \delta_{\mathrm{c}}\right)$ is the position of a tangential point $\mathrm{C}$, which is generally the center of the frame, on the celestial sphere. Furthermore, we can use the above relations in theory to estimate the corresponding error $(\mathrm{d} \xi, \mathrm{d} \eta)$ caused by its theoretical positional error $(\mathrm{d} \alpha, \mathrm{d} \delta)$, which may come from the catalogue (for example, USNO A2.0). By this, we hope to find a clear relation to simplify the reduction of observations. However, the formulae (1) and (2) are not convenient to differentiate. We may turn to their approximate relations from sphere astronomy such as Taff (1981):

$$
\begin{aligned}
& \xi=\left(\alpha-\alpha_{\mathrm{c}}\right) \cos \delta_{\mathrm{c}}-\left(\alpha-\alpha_{\mathrm{c}}\right)\left(\delta-\delta_{\mathrm{c}}\right) \sin \delta_{\mathrm{c}}+\ldots \\
& \eta=\left(\delta-\delta_{\mathrm{c}}\right)+\frac{1}{2}\left(\alpha-\alpha_{\mathrm{c}}\right)^{2} \sin \delta_{\mathrm{c}} \cos \delta_{\mathrm{c}}+\ldots
\end{aligned}
$$

Now, we have approximate relations after differentiation,

$\mathrm{d} \xi \simeq \mathrm{d} \alpha \cos \delta_{\mathrm{c}}-\mathrm{d} \alpha\left(\delta-\delta_{\mathrm{c}}\right) \sin \delta_{\mathrm{c}}-\left(\alpha-\alpha_{\mathrm{c}}\right) \sin \delta_{\mathrm{c}} \mathrm{d} \delta$

$\mathrm{d} \eta \simeq \mathrm{d} \delta+\left(\alpha-\alpha_{\mathrm{c}}\right) \sin \delta_{\mathrm{c}} \cos \delta_{\mathrm{c}} \mathrm{d} \alpha$.

Let's suppose $\delta_{\mathrm{c}}=23^{\circ}$ (in fact, our experimental objects Phoebe and a Tycho 2.0 star have $\delta_{\mathrm{c}} \simeq 23^{\circ}$, see the reference stars in Table 1), $\mathrm{d} \alpha \cos \delta_{\mathrm{c}}=\mathrm{d} \delta=0.5$ (these assumed errors are big enough even for USNO A2.0 stars), the maximum ignored error will be less than 0.5 mas for a field with the size of $400^{\prime \prime} \times 400^{\prime \prime}$ when $\mathrm{d} \xi$ and $\mathrm{d} \eta$ are replaced by $\mathrm{d} \alpha \cos \delta_{\mathrm{c}}$ and $\mathrm{d} \delta$, respectively. So, we can use the following relation for a celestial object with its initial position in some astrometric catalogue or in some ephemeris and appearing in a single CCD field of view,

$\left[\begin{array}{l}\mathrm{d} \xi \\ \mathrm{d} \eta\end{array}\right]=\left[\begin{array}{c}\mathrm{d} \alpha \cos \delta_{\mathrm{c}} \\ \mathrm{d} \delta\end{array}\right]$

It may be hard to understand the physical meaning of the relation (3). Actually for a star in a single small field of view, although the difference $\left(\xi-\Delta \alpha \cos \delta_{\mathrm{c}}, \eta-\Delta \delta\right)$ is about $S^{2} \tan \delta_{\mathrm{c}}$ (see Vienne et al. 2001b, here $S$ is the separation angle, $\Delta \alpha=\alpha-\alpha_{\mathrm{c}}$ and $\Delta \delta=\delta-\delta_{\mathrm{c}}$ ), the difference ( $\mathrm{d} \xi-\mathrm{d} \alpha \cos \delta_{\mathrm{c}}, \mathrm{d} \eta-\mathrm{d} \delta$ ) is about $S^{3} \tan \delta_{\mathrm{c}}$ since the assumed positional error $(\mathrm{d} \alpha \cos \delta, \mathrm{d} \delta)$ with the size of 0.5 can be understood as almost the same effect as $S^{2}$. So, the central projection correction is ignored in Eq. (3) but not in the computation of the standard coordinate, as we used the Eqs. (1) and (2).

On the other hand the measured standard coordinate $\left(\xi_{0}, \eta_{0}\right)$ of any celestial object in a CCD field of view has the following relation,

$\left[\begin{array}{c}\xi_{0} \\ \eta_{0}\end{array}\right]=\left[\begin{array}{cc}x-x_{\mathrm{c}} & -\left(y-y_{\mathrm{c}}\right) \\ y-y_{\mathrm{c}} & x-x_{\mathrm{c}}\end{array}\right]\left[\begin{array}{c}\rho \cos \phi \\ \rho \sin \phi\end{array}\right]$.

Where $\rho$ and $\phi$ are the scale factor and the orientation respectively of the same frame. $(x, y)$ is its pixel coordinate and $\left(x_{\mathrm{c}}, y_{\mathrm{c}}\right)$ is the center pixel coordinate of this frame. Furthermore, the difference between a celestial object's measured standard coordinate $\left(\xi_{0}, \eta_{0}\right)$ computed by Eq. (4) and its theoretical standard coordinate $\left(\xi_{\mathrm{c}}, \eta_{\mathrm{c}}\right)$ by the relations (1) and (2) is the reflectance of its theoretical positional error $\left(\mathrm{d} \alpha \cos \delta_{\mathrm{c}}, \mathrm{d} \delta\right)$ in an adopted catalogue if all random measuring errors could be ignored. In other words, we have this relation,

$\left[\begin{array}{l}\mathrm{d} \xi \\ \mathrm{d} \eta\end{array}\right]=\left[\begin{array}{c}\xi_{0}-\xi_{\mathrm{c}} \\ \eta_{0}-\eta_{\mathrm{c}}\end{array}\right]=\left[\begin{array}{ccc}x-x_{\mathrm{c}} & -\left(y-y_{\mathrm{c}}\right) & \xi_{\mathrm{c}} \\ y-y_{\mathrm{c}} & x-x_{\mathrm{c}} & \eta_{\mathrm{c}}\end{array}\right]\left[\begin{array}{c}\rho \cos \phi \\ \rho \sin \phi \\ -1\end{array}\right]$ 
When the above relation is also applied to another object in the same CCD frame we have

$\left[\begin{array}{c}\mathrm{d} \xi_{2}-\mathrm{d} \xi_{1} \\ \mathrm{~d} \eta_{2}-\mathrm{d} \eta_{1}\end{array}\right]=\left[\begin{array}{ccc}x_{2}-x_{1} & -\left(y_{2}-y_{1}\right) & \xi_{2 \mathrm{c}}-\xi_{1 \mathrm{c}} \\ y_{2}-y_{1} & x_{2}-x_{1} & \eta_{2 \mathrm{c}}-\eta_{1 \mathrm{c}}\end{array}\right]\left[\begin{array}{c}\rho \cos \phi \\ \rho \sin \phi \\ -1\end{array}\right]$

According to the relation (3), the above relation can be further changed into,

$\left[\begin{array}{c}\mathrm{d} \alpha_{2}-\mathrm{d} \alpha_{1} \\ \mathrm{~d} \delta_{2}-\mathrm{d} \delta_{1}\end{array}\right]=\left[\begin{array}{ccc}\Delta x^{\prime} & -\Delta y^{\prime} & \Delta \xi \\ \Delta y & \Delta x & \Delta \eta\end{array}\right]\left[\begin{array}{c}\rho \cos \phi \\ \rho \sin \phi \\ -1\end{array}\right]$

Where,

$$
\left\{\begin{aligned}
\Delta x & =x_{2}-x_{1}, \\
\Delta y & =y_{2}-y_{1} \\
\Delta \eta & =\eta_{2 \mathrm{c}}-\eta_{1 \mathrm{c}} \\
\Delta x^{\prime} & =\Delta x / \cos \delta_{\mathrm{c}} \\
\Delta y^{\prime} & =\Delta y / \cos \delta_{\mathrm{c}} \\
\Delta \xi & =\left(\xi_{2 \mathrm{c}}-\xi_{1 \mathrm{c}}\right) / \cos \delta_{\mathrm{c}}
\end{aligned}\right.
$$

\subsection{Overlap exposure method}

In Fig. 1, $\mathrm{S}_{\mathrm{I}}$ and $\mathrm{S}_{\mathrm{II}}$ are two reference stars with errors supposed to be zero (see assumption ii)). $\mathrm{N}$ frames of overlapped CCD images in which a target object is contained cover the whole celestial area between the two reference stars. A small shift (usually several minutes of a degree) between any two neighborhood CCD frames is designed to assure that one connection star (or satellite) appears, that is, the requirement iii) is satisfied.

In the first field of view $\mathrm{F}_{1}$ (in Fig. 1), an error ( $\left.\mathrm{d} \alpha_{1}, \mathrm{~d} \delta_{1}\right)$ in the theoretical position of $S_{1}$ in the right ascension and in declination can be denoted with high precision by the following relation when it is measured with respect to $S_{I}$ according to the relation $(5)$.

$\left[\begin{array}{c}\mathrm{d} \alpha_{1} \\ \mathrm{~d} \delta_{1}\end{array}\right]=\left[\begin{array}{ccc}\Delta x_{1}^{\prime} & -\Delta y_{1}^{\prime} & \Delta \xi_{1} \\ \Delta y_{1} & \Delta x_{1} & \Delta \eta_{1}\end{array}\right]\left[\begin{array}{c}\rho \cos \phi \\ \rho \sin \phi \\ -1\end{array}\right]$

Where,

$$
\left\{\begin{array}{l}
\Delta x_{1}=x_{1,1}-x_{\mathrm{I}, 1}, \\
\Delta y_{1}=y_{1,1}-y_{\mathrm{I}, 1}, \\
\Delta \eta_{1}=\eta_{1 \mathrm{c}_{1}}-\eta_{\mathrm{Ic}_{1}}, \\
\Delta x_{1}^{\prime}=\Delta x_{1} / \cos \delta_{\mathrm{c}_{1}}, \\
\Delta y_{1}^{\prime}=\Delta y_{1} / \cos \delta_{\mathrm{c}_{1}}, \\
\Delta \xi_{1}=\left(\xi_{1 \mathrm{c}_{1}}-\xi_{\mathrm{Ic}_{1}}\right) / \cos \delta_{\mathrm{c}_{1}} .
\end{array}\right.
$$

In detail, $\left(x_{1,1}, y_{1,1}\right),\left(x_{\mathrm{I}, 1}, y_{\mathrm{I}, 1}\right)$ are the raw pixel coordinates of the two objects $S_{1}$ and $S_{I}$, respectively, and $\left(\xi_{1 c_{1}}, \eta_{1 c_{1}}\right)$, $\left(\xi_{\mathrm{Ic}_{1}}, \eta_{\mathrm{Ic}_{1}}\right)$ are their corresponding standard coordinates which are determined according to Eqs. (1) and (2) using their theoretical positions and center equatorial coordinates $\left(\alpha_{\mathrm{c}_{1}}, \delta_{\mathrm{c}_{1}}\right)$ in the same frame $F_{1}$.

In the second frame $F_{2}$ in Fig. $1, S_{1}$ and a new connection star $\mathrm{S}_{2}$ appear. Similarly, an error $\left(\mathrm{d} \alpha_{2}, \mathrm{~d} \delta_{2}\right)$ in the theoretical

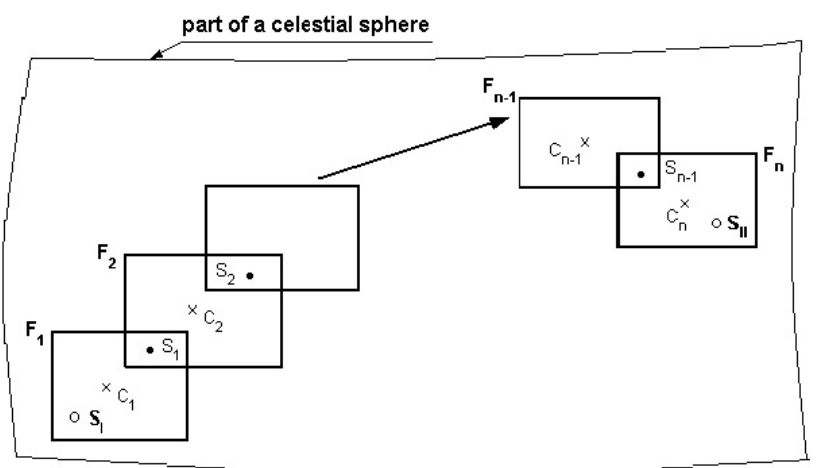

Fig. 1. A sketch of $\mathrm{n}$ overlapped frames of CCD images. $\mathrm{F}_{1}, \mathrm{~F}_{2}, \ldots, \mathrm{F}_{n}$ are $n$ combined fields of view. $S_{\mathrm{I}}$ and $\mathrm{S}_{\mathrm{II}}$ are the reference stars, and $\mathrm{S}_{1}, \mathrm{~S}_{2}, \ldots, \mathrm{S}_{n-1}$ are the connection stars (see Sect. 2.1).

position of $S_{2}$ when measured with respect to $S_{1}$ in right ascension and in declination can be shown as follows:

$\left[\begin{array}{c}\mathrm{d} \alpha_{2}-\mathrm{d} \alpha_{1} \\ \mathrm{~d} \delta_{2}-\mathrm{d} \delta_{1}\end{array}\right]=\left[\begin{array}{ccc}\Delta x_{2}^{\prime} & -\Delta y_{2}^{\prime} & \Delta \xi_{2} \\ \Delta y_{2} & \Delta x_{2} & \Delta \eta_{2}\end{array}\right]\left[\begin{array}{c}\rho \cos \phi \\ \rho \sin \phi \\ -1\end{array}\right]$

Where

$$
\left\{\begin{array}{l}
\Delta x_{2}=x_{2,2}-x_{1,2}, \\
\Delta y_{2}=y_{2,2}-y_{1,2}, \\
\Delta \eta_{2}=\eta_{2 \mathrm{c}_{2}}-\eta_{1 \mathrm{c}_{2}}, \\
\Delta x_{2}^{\prime}=\Delta x_{2} / \cos \delta_{\mathrm{c}_{2}}, \\
\Delta y_{2}^{\prime}=\Delta y_{2} / \cos \delta_{\mathrm{c}_{2}}, \\
\Delta \xi_{2}=\left(\xi_{2 \mathrm{c}_{2}}-\xi_{1 \mathrm{c}_{2}}\right) / \cos \delta_{\mathrm{c}_{2}} .
\end{array}\right.
$$

Here, $\left(x_{1,2}, y_{1,2}\right),\left(x_{2,2}, y_{2,2}\right)$ are the raw pixel coordinates of the two objects $S_{1}$ and $S_{2}$, respectively, in the second field of view $\mathrm{F}_{2}$, and $\left(\xi_{1 \mathrm{c}_{2}}, \eta_{1 \mathrm{c}_{2}}\right),\left(\xi_{2 \mathrm{c}_{2}}, \eta_{2 \mathrm{c}_{2}}\right)$ are their respective standard coordinates in the same frame. It should be noted that the standard coordinates of $S_{1}$ in the first field of view $F_{1}$ and in the second field of view $\mathrm{F}_{2}$ are completely different since the equatorial coordinates of the two tangential points $\mathrm{C}_{1}, \mathrm{C}_{2}$ in $\mathrm{F}_{1}$ and $\mathrm{F}_{2}$ are obviously different. On the other hand, the positional error of $S_{2}$ with respect to the reference star $S_{I}$ can be shown as the following relation since all stars $S_{1}, S_{2}$, and $S_{I}$ can also be thought to be on the same celestial sphere and after the relations (6) and (7) are considered:

$\left[\begin{array}{l}\mathrm{d} \alpha_{2} \\ \mathrm{~d} \delta_{2}\end{array}\right]=\left[\begin{array}{ccc}\sum_{j=1}^{2} \Delta x_{j}^{\prime} & -\sum_{j=1}^{2} \Delta y_{j}^{\prime} & \sum_{j=1}^{2} \Delta \xi_{j} \\ \sum_{j=1}^{2} \Delta y_{j} & \sum_{j=1}^{2} \Delta x_{j} & \sum_{j=1}^{2} \Delta \eta_{j}\end{array}\right]\left[\begin{array}{c}\rho \cos \phi \\ \rho \sin \phi \\ -1\end{array}\right]$.

Furthermore, a theoretical positional error of the connection star $S_{i}$ in the $i$ th field of view $F_{i}$ with respect to $S_{\mathrm{I}}$ in the first field of view $F_{1}$ can be derived into the following relation,

$\left[\begin{array}{c}\mathrm{d} \alpha_{i} \\ \mathrm{~d} \delta_{i}\end{array}\right]=\left[\begin{array}{ccc}\sum_{j=1}^{i} \Delta x_{j}^{\prime} & -\sum_{j=1}^{i} \Delta y_{j}^{\prime} & \sum_{j=1}^{i} \Delta \xi_{j} \\ \sum_{j=1}^{i} \Delta y_{j} & \sum_{j=1}^{i} \Delta x_{j} & \sum_{j=1}^{i} \Delta \eta_{j}\end{array}\right]\left[\begin{array}{c}\rho \cos \phi \\ \rho \sin \phi \\ -1\end{array}\right]$. 
Lastly, the theoretically positional error of $S_{\text {II }}$ with respect to $S_{I}$ after $n$ overlapped frames of CCD images are taken would have the following relation,

$\left[\begin{array}{l}\mathrm{d} \alpha_{\mathrm{II}} \\ \mathrm{d} \delta_{\mathrm{II}}\end{array}\right]=\left[\begin{array}{ccc}\sum_{j=1}^{n} \Delta x_{j}^{\prime} & -\sum_{j=1}^{n} \Delta y_{j}^{\prime} & \sum_{j=1}^{n} \Delta \xi_{j} \\ \sum_{j=1}^{n} \Delta y_{j} & \sum_{j=1}^{n} \Delta x_{j} & \sum_{j=1}^{n} \Delta \eta_{j}\end{array}\right]\left[\begin{array}{c}\rho \cos \phi \\ \rho \sin \phi \\ -1\end{array}\right]$.

According to the assumption ii) ( $\left.\mathrm{d} \alpha_{\mathrm{II}}=0, \mathrm{~d} \delta_{\mathrm{II}}=0\right)$, the calibration parameters can be solved from the above Eq. (10),

$$
\begin{aligned}
\rho \cos \phi & =\frac{\sum_{j=1}^{n} \Delta \xi_{j} \sum_{j=1}^{n} \Delta x_{j}+\sum_{j=1}^{n} \Delta \eta_{j} \sum_{j=1}^{n} \Delta y_{j}^{\prime}}{\sum_{j=1}^{n} \Delta x_{j} \sum_{j=1}^{n} \Delta x_{j}^{\prime}+\sum_{j=1}^{n} \Delta y_{j} \sum_{j=1}^{n} \Delta y_{j}^{\prime}} \\
\rho \sin \phi & =\frac{-\sum_{j=1}^{n} \Delta \xi_{j} \sum_{j=1}^{n} \Delta y_{j}+\sum_{j=1}^{n} \Delta \eta_{j} \sum_{j=1}^{n} \Delta x_{j}^{\prime}}{\sum_{j=1}^{n} \Delta x_{j} \sum_{j=1}^{n} \Delta x_{j}^{\prime}+\sum_{j=1}^{n} \Delta y_{j} \sum_{j=1}^{n} \Delta y_{j}^{\prime}} .
\end{aligned}
$$

After the Eqs. (11) and (12) are solved, the theoretical positional errors for any connection star or target object in the 1st, 2nd and ith field of view can be solved according to the Eqs. (6), (8) and (9) respectively with respect to $S_{I}$ (as an option, these positional errors can also be determined with respect to the $\mathrm{S}_{\mathrm{II}}$ ). During the practical application of the method, the topocentric equatorial coordinates of the reference stars $S_{I}, S_{I I}$ and all connection stars $S_{1}, S_{2}, \cdots, S_{n-1}$ are required in their computation. Atmospheric refractions also need to be taken into account. Last, the equatorial coordinate of the tangential point in each frame can be solved iteratively as done by Vienne et al. (2001a) and Peng et al. (2002) for the reduction of a single frame of a CCD image until the solution of the two calibration parameters, scale and orientation, converge.

\section{Experimental observations}

$50 \mathrm{CCD}$ images in total for the 9th satellite, Phoebe, and 27 CCD images for the tested bright star (in the Tycho 2.0 catalogue) were obtained with the 1-m telescope at the Yunnan Observatory on the nights of Feb. 18, 27 and 28, 2003. While observing, a Johnson-I type filter was used for each frame of the CCD image, and exposure times of $40 \sim 80 \mathrm{~s}$ were adopted depending on the atmospheric conditions. The specifications of the telescope used and the CCD receiver are given in Table 1 in Peng et al. (2002). Due to the slow motion of Phoebe, CCD overlap exposures could be performed in the same celestial area for three nights' observations. The two reference used stars, ACT1 and ACT2 were extracted from the ACT catalogue (Urban et al. 2001) and the 2 connection stars $S_{1}$ and $S_{2}$ from the USNO A2.0 catalogue (Monet et al. 1998). The tested bright star T2 (see Fig. 2a) can be found in the Tycho 2.0 catalogue (Høg et al. 2000) although it cannot be found in the ACT catalogue. The detailed data items extracted from the corresponding catalogues for all stars are listed in Table 1. Figure 2 shows one series of calibrated CCD images obtained on the night of Feb. 18, 2003. Two reference stars ACT1 and ACT2 appear in the first and third field of view $F_{1}$ and $F_{3}$, respectively. We see also from Figs. $2 \mathrm{a}$ and $\mathrm{b}$ that Phoebe is very faint ( $V \sim 16.3 \mathrm{mag})$.
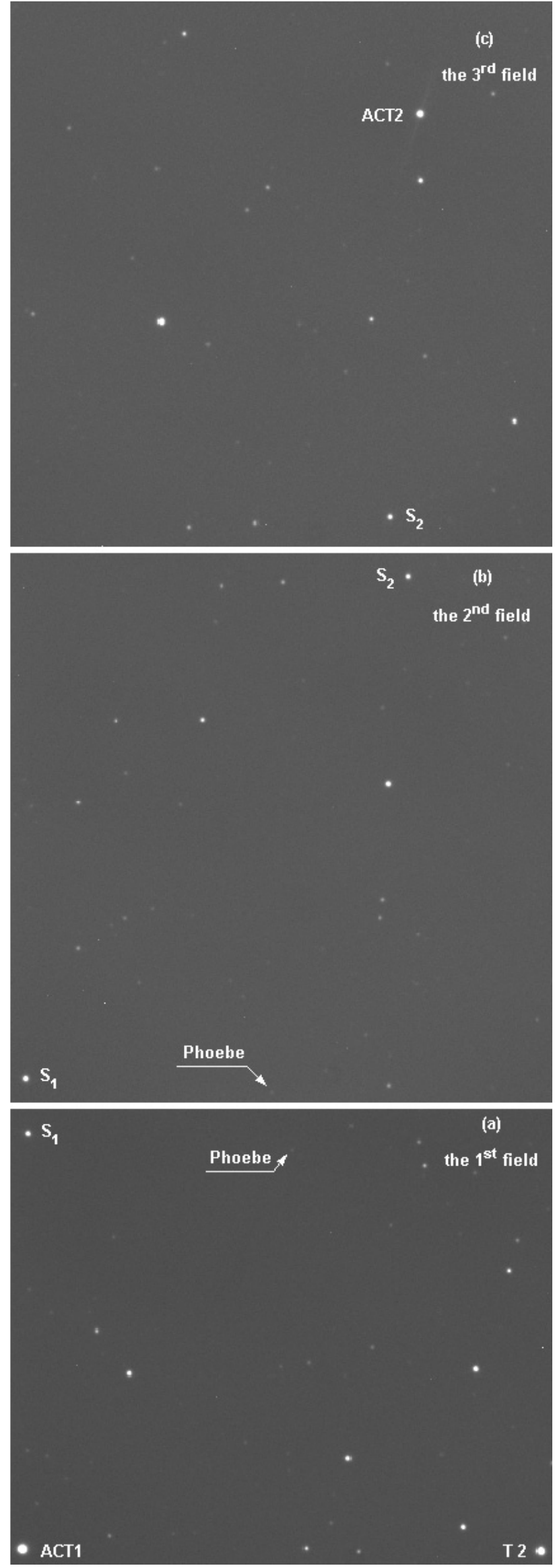

Fig. 2. Three typical overlapped CCD images. 
Table 1. Reference stars, tested star and connected stars from ACT, Tycho 2.0 and USNO A2.0, respectively. ID is the identification used in this paper, No. means the star identification number in the corresponding catalogue. The star identification numbers and proper motions for connection stars are not available in USNO A2.0, and the value " 0 " is adopted for their proper motions.

\begin{tabular}{|c|c|c|c|c|c|c|c|}
\hline ID & Cat & No. & RA (J2000) & Dec (J2000) & $\mu_{\alpha}$ & $\mu_{\delta}$ & Magnitude ( $V$-mag) \\
\hline ACT1 & ACT & 645062 & $05^{\mathrm{h}} 28^{\mathrm{m}} 31^{\mathrm{s}} .7318$ & $+22^{\circ} 06^{\prime} 34^{\prime \prime} \cdot 705$ & $-0.00149 / \mathrm{yr}$ & $-0.0065 / \mathrm{yr}$ & 10.545 \\
\hline ACT2 & ACT & 645018 & $05^{\mathrm{h}} 28^{\mathrm{m}} 13^{\mathrm{s}} .3216$ & $+22^{\circ} 19^{\prime} 36^{\prime \prime} \cdot 174$ & $-0.00026 / \mathrm{yr}$ & $0.0008 / \mathrm{yr}$ & 11.610 \\
\hline $\mathrm{T} 2$ & Tycho 2.0 & 130902662 & $05^{\mathrm{h}} 28^{\mathrm{m}} 10^{\mathrm{s}} .3574$ & $+22^{\circ} 06^{\prime} 33^{\prime \prime} .813$ & $0 \varsigma 00024 / \mathrm{yr}$ & $-0{ }^{\prime} 0035 / \mathrm{yr}$ & 11.238 \\
\hline $\mathrm{S}_{1}$ & USNO A2.0 & $* *$ & $05^{\mathrm{h}} 28^{\mathrm{m}} 31^{\mathrm{s}} .5467$ & $+22^{\circ} 10^{\prime} 33^{\prime \prime} .500$ & $0^{*}$ & $0^{*}$ & 13.100 \\
\hline $\mathrm{S}_{2}$ & USNO A2.0 & $* *$ & $05^{\mathrm{h}} 28^{\mathrm{m}} 14.5620$ & $+22^{\circ} 15^{\prime} 44^{\prime \prime} .010$ & $0 *$ & $0 *$ & 13.900 \\
\hline
\end{tabular}

\section{Calibration}

Before calibration, we measured all raw pixel coordinates of stars and Phoebe using a modified moment method, which has been applied to some major Saturnian satellites and Galilean satellites (Peng et al. 2002, 2003). Although two calibration parameters - the scale $\rho$ and orientation $\phi$ are widely used to describe the astrometric calibration of a CCD image, we find here that the forms of $\rho \cos \phi$ and $\rho \sin \phi$ for the two calibration parameters are even more convenient for our new method. Table 2 shows these parameters determined on three nights.

\section{Results}

\subsection{Positions of $T 2$ with respect to ACT1}

To certify the calibration parameters on each observational date, we determine the position of the star T2 in the 1st field of view (see Fig. 2a) when measured with respect to ACT1. In theory, the mean positional errors $(\mathrm{O}-\mathrm{C})$ (Observed minus Computed) of $\mathrm{T} 2$ should be constant for all observations over a short period of time. Table 3 shows the expected results with small scatter. There are two types of observations on Feb. 18 in Table 3. The listed one ( 5 observations) is obtained in the 3 -frame observations used to calibrate; the other (10 observations among 16 images since $\mathrm{T} 2$ is out of the frame in the other 6 images) is obtained in a single frame before calibration observations. Therefore, the calibration parameters for the observations in a single frame have to be adopted from calibrated observations. The same applies for the following observations of Phoebe in Table 4.

\subsection{Positions of Phoebe with respect to ACT1 and comparison with other CCD observations}

As seen from Figs. 2a and b, Phoebe appears in the 1st frame and the 2nd one. This provides more opportunities to measure its position in two frames. When it appears in the 1st one, its position can be directly determined with respect to ACT1, and we call it a "Direct Measure". On the other hand, when it appears in the 2 nd frame, its position can be first determined with respect to $S_{1}$, then transformed into its positional measurement with respect to ACT1 by $S_{1}$ in the 1 st frame. We call this an "Indirect Measure". Table 4 shows the positional results from both the "Direct Measure" and "Indirect Measure" for Phoebe, whose geocentric apparent position is obtained according to
Table 2. Solved calibration parameters (unit: $\operatorname{arcsec} /$ pixel). $N$ denotes the number of overlapping exposure series. $\langle\rho \cos \phi\rangle$ and $\langle\rho \sin \phi\rangle$ are the mean values for the calibration parameters, and SD is the standard deviation.

\begin{tabular}{cccccc}
\hline \hline Date & $N$ & $\langle\rho \cos \phi\rangle$ & SD & $\langle\rho \sin \phi\rangle$ & SD \\
\hline 02.18 & 5 & -.373786 & .000022 & .000442 & .000011 \\
02.27 & 3 & -.373772 & .000021 & .003576 & .000005 \\
02.28 & 9 & -.373767 & .000015 & .003580 & .000010 \\
\hline
\end{tabular}

Table 3. Positional measurement of the tested star $\mathrm{T} 2$ with respect to ACT1 (unit: arcsec). $N$ denotes the number of observations. $\langle\triangle \alpha \cos \delta\rangle$ and $\langle\Delta \delta\rangle$ are the mean (O-C)s in right ascension and in declination respectively, and SD is the standard deviation.

\begin{tabular}{cccccc}
\hline \hline Date & $N$ & $\langle\Delta \alpha \cos \delta\rangle$ & $\mathrm{SD}$ & $\langle\Delta \delta\rangle$ & $\mathrm{SD}$ \\
\hline 02.18 & $10^{*}$ & -.005 & .006 & -.019 & .013 \\
02.18 & 5 & -.019 & .010 & .004 & .014 \\
02.27 & 3 & -.033 & .013 & -.013 & .020 \\
02.28 & 9 & -.007 & .017 & -.018 & .020 \\
\hline Total & $N$ & $\langle\Delta \alpha \cos \delta\rangle$ & $\mathrm{SD}$ & $\langle\Delta \delta\rangle$ & $\mathrm{SD}$ \\
\hline & 27 & -.011 & .015 & -.014 & .018 \\
\hline
\end{tabular}

the models JPL DE406 and SAT136 via the internet web site (http://ssd.jpl.nasa.gov/). Atmospheric refraction, diurnal parallax and diurnal aberration are all taken into account.

As shown in Table 4, direct measurement usually has better precision than the indirect one. All observations on different dates have a quite good agreement. The global precision is about \pm 0.07 arcsec in right ascension and in declination for a single observation. Slightly poorer quality on the night of Feb. 27, 2003 results mainly from poor atmospheric conditions. When we compare the results in Table 4 with those in Table 3, we find that Phoebe has far worse quality in its positional measurement than T2. The main cause is its faint brightness. Also, indirect measurement is partly responsible for the poor quality since the measuring error of $S_{1}$ is incorporated into the position of Phoebe. When compared to recently published CCD observations in Veiga et al. (2000) and Fienga et al. (2002) as shown in Table 5, the new calibration method allows us to obtain a "clean" and more precise position of Phoebe since our observation is obtained directly from the ACT catalogue. SAT136 in our reduction is the latest ephemeris that has been developed for "CASSINI" and it is thought to be 
Table 4. Mean $(\mathrm{O}-\mathrm{C}) \mathrm{s}(\langle\Delta \alpha \cos \delta\rangle,\langle\Delta \delta\rangle)$ (unit: $\operatorname{arcsec}$ ) for positional measurement of Phoebe with respect to ACT1. $N$ denotes the number of observations. SD is the standard deviation.

\begin{tabular}{|c|c|c|c|c|c|}
\hline Direct & & Measure & & & \\
\hline Date & $N$ & $\langle\Delta \alpha \cos \delta\rangle$ & SD & $\langle\Delta \delta\rangle$ & SD \\
\hline 02.18 & $16^{*}$ & .019 & .040 & -.096 & .048 \\
\hline 02.18 & 5 & -.035 & .036 & -.111 & .031 \\
\hline 02.27 & 3 & -.001 & .126 & -.060 & .061 \\
\hline 02.28 & 9 & -.026 & .051 & -.049 & .060 \\
\hline Indirect & & Measure & & & \\
\hline Date & $N$ & $\langle\Delta \alpha \cos \delta\rangle$ & SD & $\langle\Delta \delta\rangle$ & SD \\
\hline 02.18 & 5 & -.021 & .074 & -.077 & .084 \\
\hline 02.27 & 3 & .050 & .101 & .014 & .096 \\
\hline 02.28 & 9 & -.056 & .070 & -.066 & .047 \\
\hline \multirow[t]{3}{*}{ Total } & & Measure & & & \\
\hline & $N$ & $\langle\Delta \alpha \cos \delta\rangle$ & SD & $\langle\Delta \delta\rangle$ & $\mathrm{SD}$ \\
\hline & 50 & -.011 & .068 & -.073 & .061 \\
\hline
\end{tabular}

Table 5. Comparison with recently published CCD observations (unit: arcsec) $(\mathrm{V}+00$ : Veiga et al. 2000, F+02: Fienga et al. 2002) when measured with respect to the theory of Jacobson (1998). $N$ denotes the number of observations. $\langle\Delta \alpha \cos \delta\rangle$ and $\langle\Delta \delta\rangle$ are the mean $(\mathrm{O}-\mathrm{C}) \mathrm{s}$ in right ascension and declination respectively, and SD is the standard deviation.

\begin{tabular}{cccccc}
\hline \hline Obs & $N$ & $\langle\Delta \alpha \cos \delta\rangle$ & SD & $\langle\Delta \delta\rangle$ & SD \\
\hline V+00 & 60 & -.080 & .140 & .290 & .260 \\
F+02 & 163 & .156 & .148 & .154 & .177 \\
The work & 50 & -.011 & .068 & -.073 & .061 \\
\hline
\end{tabular}

considerably more accurate than the 1998 ephemeris (Jacobson 1998). Our results suggest a significant improvement in accuracy for the ephemeris SAT136 although more observations are needed. The comparison shown in Table 5 is somewhat underestimated for recently published observations since some small errors in its orbit theory of the satellite would have extra effects on their estimated formal errors.

\section{Conclusions}

A series of overlapped CCD images, obtained on a small celestial area that encloses the target object, can be used to determine precisely the calibration parameters in the same area. In theory, the area must be as small as possible. The accuracy of the obtained calibration parameters $\rho$ and $\phi$ (or equivalently, $\rho \cos \phi$ and $\rho \sin \phi$ ) depend on the mechanical properties of the telescope used and/or the size of the observed celestial area. The proposed method has been proven to be good enough to determine precise positions of Phoebe as well as a tested bright star. Some small theoretical positional errors of reference stars enter the solved calibration parameters. Therefore, more reference stars with good theoretical positions should be found based on a statistical method. Lastly, the overlap method could also be applicable to astrometric observations for other natural satellites of major planets, asteroids and optical counterparts of extragalactic radio sources when the highly dense distribution of stars in some contemporary astrometric catalogues such as ACT and Tycho 2.0 is taken into account.

Acknowledgements. It was a pleasure for the first author to acknowledge Drs Arlot, Thuillot and Lainey in the IMCCE, and Dr. Jacobson in the JPL for their valuable discussions and helpful suggestions. This work was carried out with financial support from the PHEMU03 international campaign organized by the IMCCE and from the National Natural Science Foundation of China (Grant No. 10273015).

\section{References}

Colas, F., \& Arlot, J. E. 1991, A\&A, 252, 402

Fienga, A., Arlot, J. E., Baron, N., et al. 2002, A\&A, 391, 767

Harper, D., Murray, C. D., Beurle, K., et al. 1997, A\&AS, 121, 65

Høg, E., Fabricius, C., Makarov, V. V., et al. 2000, A\&A, 355, L27

Jacobson, R. A. 1998, A\&AS, 128, 7

Monet, D., Bird, A., Canzian, B., et al. 1998, The USNO-A2.0 Catalogue (Washington, DC: US Naval Observatory)

Pascu, D. 1996, IAU symp., 172, 373

Peng, Q. Y., Vienne, A., \& Shen, K. X. 2002, A\&A, 383, 296

Peng, Q. Y., Han, Y. B., Zhang, C. L., et al. 2003, A\&A, 401, 773

Shen, K. X., Dourneau, G., Qiao, R. C., et al. 2001, A\&A, 367, 1061

Stone, R. C., \& Harris, F. H. 2000, AJ, 119, 1985

Taff, L. G. 1981, Computational Spherical Astronomy (John Wiley \& Sons Inc.)

Urban, S. E., Corbin, T. E., \& Wycoff, G. L. 1998, AJ, 115, 2161

Veiga, C. H., \& Vieira, M. R. 1994, A\&AS, 107, 551

Veiga, C. H., Vieira, M. R., \& Andrei, A. H. 2000, A\&AS, 142, 81

Veiga, C. H., Vieira, M. R., Vienne, A., et al. 2003, A\&A, 400, 1095

Vienne, A., Thuillot, W., Veiga, C. H., et al. 2001a, A\&A, 380, 727

Vienne, A., Thuillot, W., \& Arlot, J. E. 2001b, Note Scientifique et Technique, de l'IMCCE, S077 